\title{
Quantitation of microRNA-92a in colorectal adenocarcinoma and its precancerous lesions: Co-utilization of in situ hybridization and spectral imaging
}

\author{
I WENG LAO ${ }^{1}$, FENGYUN CUI $^{1}$ and HONGGUANG ZHU ${ }^{1-3}$ \\ ${ }^{1}$ Department of Pathology, School of Basic Medical Sciences; ${ }^{2}$ Division of Surgical Pathology, \\ Huashan Hospital; ${ }^{3}$ Institutes of Biomedical Sciences, Fudan University, Shanghai 200032, P.R. China \\ Received February 9, 2014; Accepted October 31, 2014
}

DOI: $10.3892 / \mathrm{ol} .2014 .2813$

\begin{abstract}
The expression level of microRNA (miR)-92a has been proven to increase during the development of colorectal adenocarcinoma (CA) and has been verified at the cellular, plasma and fecal levels by various quantitative methods. However, a method to quantitate the expression level using tissue sections has not been established. To do this, in situ hybridization (ISH) and multispectral imaging microscopy (MSI) were introduced to quantitate miR-92a expression on the microscopic level. ISH of miR-92a was first performed on 34 tissue samples of CA and adenomas with high-grade and low-grade intraepithelial neoplasms, while 31 paralesional normal tissue samples were defined as the control. Subsequently, a MSI technique was applied to quantitate the hybridization signal in terms of optical density (OD) at the visible wavelength. A t-test with unequal variance was used to examine the statistical significance between the groups. Despite all 34 tissue sections demonstrating at least partial positivity of miR-92a expression following ISH, visual grading was inconclusive. As such, the signal of ISH was transformed in terms of OD and further analyzed by employing the MSI system. A statistically significant difference was observed between the expression levels of miR-92a in CA and the paralesional normal controls. By contrast, a poor correlation was revealed between visual and spectral grading. The co-utilization of ISH and MSI generated a legible observation in the expression level of miR-92a, revealing the dynamic change in miR-92a expression in the progression of the disease and providing important information for further functional investigation.
\end{abstract}

\section{Introduction}

microRNA (miRNA/miR) is a 22 nt long, non-coding small RNA that is essential in post-transcriptional regulation and

Correspondence to: Professor Hongguang Zhu, Department of Pathology, School of Basic Medical Sciences, Fudan University, 138 Yixueyuan Road, Shanghai 200032, P.R. China

E-mail: hongguang_702@163.com

Key words: in situ hybridization, multispectral imaging microscopy, colorectal adenocarcinoma, microRNA, miR-92a, quantitation has been suggested to directly and indirectly regulate genesis, differentiation, proliferation, growth and apoptosis in eukaryotes, particularly in carcinogenesis (1-4).

As one well-established technique used to quantitatively study micromolecules, in situ hybridization (ISH) is favored due to its ability to directly observe the spatial expression of the studied candidate. Through utilizing radioactive isotopes, fluorophores or chromophores as indicators to label any single complementary DNA or RNA strand so that the strand serves as a probe and targets specific counterstrands of interest, ISH allows the visualization of minute changes within cells $(5,6)$. Variant forms of ISH have been derived and widely applied in the field of diagnostics, as well as in fundamental studies (6).

Multispectral imaging microscopy (MSI) is an advanced method applied in the analysis of macro- and microscopic samples from a three-dimensional aspect $(7,8)$. MSI is a quantitative technique that adds spatial resolution to the spectral images when analyzing samples, first performed by assigning intensity as a function of wavelength, then by acquiring an image as a constellation of pixel units, with each pixel unit classified by its spectral signature, and genuinely creating an image cube that contains spectral and spatial information $(8,9)$. This does not only resolve the expression of numerous components within a single cell, but also generates information on any dynamic changes from normal to aberrant cells, providing a simple yet convenient method for biomedical studies (9-11).

The present study attempted to observe the changes in the expression level of miR-92a at a tissue level, as miR-92a had been previously proven to increase at the cellular, plasma and fecal levels during the development of colorectal adenocarcinoma (CA) (12-14). It was found that quantitation of miRNAs was not realized by ISH independently, as miR-92a was only expressed in cells with a differentiation in level, which was consistent with the findings of Liang et al (15). Therefore, a secondary approach of employing a spectral imaging technique followed by ISH was developed in an attempt to quantitate the changes in level of miR-92a expression.

\section{Materials and methods}

Tissue samples. In total, 34 tissue samples of colorectal lesions collected from the surgically resected specimens 
obtained from colorectal cancer patients who underwent hemicolorectomies and colorectomies at Huashan Hospital (Shanghai, China) between 2009 and 2012, that represented three consecutive stages were grouped as follows: 10 samples of low-grade intraepithelial neoplasia (LGIN), 11 samples of high-grade intraepithelial neoplasia (HGIN) and 13 samples of CA. For the internal normal control, 31 normal paralesional tissue samples were obtained, and were divided into three groups: Nine normal controls for LGIN (LGIN-N), 10 for HGIN (HGIN-N) and 12 for CA (CA-N). Three samples possessed no adjacent normal mucosa samples. Diagnoses were confirmed by two senior pathologists in the Department of Pathology (School of Basic Medical Sciences, Fudan University, Shanghai, China). The study was approved by the ethics committee of Shanghai Medical College, Fudan University. Written informed consent was obtained from all patients.

miRNA in situ hybridization. All samples were fixed with $10 \%$ buffered formalin and were paraffin embedded. Each sample block was sectioned into $6-\mu \mathrm{m}$ thick slices and mounted on charged slides. Hybridization was performed following the procedures reported previously $(16,17)$. Briefly, the slides were deparaffinized and dehydrated with xylene and an ascending gradient of ethanol and the slides were then rehydrated using phosphate-buffered saline (PBS) at pH 7.4. Subsequently, proteinase digestion was performed using proteinase $\mathrm{K}(15 \mu \mathrm{g} / \mathrm{ml}$; Exiqon, Vedbaek, Denmark) for $8 \mathrm{~min}$ at $37^{\circ} \mathrm{C}$, and the slides were washed with $3 \mathrm{X}$ PBS at pH 7.4. Hybridization was performed with a locked nucleic acid (LNA)-modified, 5'-digoxigenin-labeled probe of miR-92a (sense, 5'-ACAGGCCGGGACAAGTGCAATA-3'; Exiqon) at a concentration of $40 \mathrm{nM}$, using PTC-100 ${ }^{\mathrm{TM}}$ Programmable Thermal Controller (MJ Research, Inc., Waltham, MA, USA) at $55^{\circ} \mathrm{C}$ for $1 \mathrm{~h}$. Following hybridization, a stringency wash was performed on the slides with a descending gradient of saline-sodium citrate (5X, $2 \mathrm{X}$ and $0.2 \mathrm{X})$ at $4^{\circ} \mathrm{C}(17)$. Blocking was performed using anti-digoxigenin alkaline-phosphatase combined with sheep serum (DIG Nucleic Acid Detection kit; Roche Diagnostics, Indianapolis, IN, USA) at room temperature for $1 \mathrm{~h}$. The slides were then stained with nitro blue tetrazolium/5-bromo-4-chloro-3-indolyl phosphate (NBT/BCIP; SIGMAFAST ${ }^{\mathrm{TM}}$ BCIP $^{\circledR} /$ NBT; Sigma-Aldrich, St. Louis, MO, USA) for signal development, and the nuclei were counterstained with methyl green.

Visual grading of ISH result. The average expression of miR-92a on the slides was visually and individually graded by two pathologists using a light microscope at x200 magnification (Carl Zeiss Microscopy GmbH, Göttingen, Germany). A four-tier scoring system was devised according to the cytoplasmic staining intensity: i) Negative, unstained cytoplasm or cytoplasm exhibiting only background color; ii) weak positive, cytoplasm exhibiting a light indigo color; iii) moderate, cytoplasm exhibiting a weak to moderate indigo stain; and iv) strong positive, cytoplasm exhibiting a dark indigo stain.

Image acquisition and signal interpretation by MSI system. Using the CRi-Nuance ${ }^{\mathrm{TM}}$ Multispectral Imaging System
(Cambridge Research and Instrumentation Inc., Woburn, MA, USA), one representative unstained slide from each of the three investigated groups was selected and stained with NBT/BCIP and methyl green, respectively. These slides were used to obtain spectral references, as shown in Fig. 1. For each tissue sample, a region of interest (ROI) demonstrating the average visual grading was randomly selected within the site of miR-92a expression, and images were then acquired under x200 magnification using a charge-coupled device (CCD) camera to accompany the imaging system. The average NBT/BCIP optical density (OD) signal was detected from each field, and the average signal and pixel areas were further generated within the ROI by the Nuance analyzer (Cambridge Research and Instrumentation Inc.) as shown in Fig. 2. Signal interpretation was also devised into a four-tiered system by a quartile cut-off value according to the minimum $(0.20825)$ and maximum (0.9455) OD: i) Negative, OD $\leq 0.377$; ii) weak positive, OD 0.378-0.5105; iii) moderate, OD 0.5106-0.68025; and iv) strong positive, OD $>0.68025$. The average signal was calculated from the area using unit pixels and the concentration of miR-92a in the cytoplasm, as represented by the NBT/BCIP concentration, as follows: Average signal $=$ Total signal $/$ Area pixels. Provided that miR-92a concentration per cell $=$ total NBT / BCIP concentration per cell, miR-92a concentration per cell $=$ miR-92a concentration within (cytoplasm + nucleus): NBT/BCIP cytoplasm = [total signal (full image) - total signal (overlap)] / [total signal (full image) - total signal (overlap)]. Thus, miR-92a concentration within the cytoplasm = total NBT/BCIP concentration per cell - NBT/BCIP concentration within the nucleus, which was therefore, the concentration of NBT/BCIP in the cytoplasm, the non-overlapped area with NBT/BCIP expression per cell.

Statistical analysis. Using Microsoft Excel 2007 (Microsoft, Redmond, WA, USA), a t-test with unequal variance was used to compare LGIN, HGIN, CA and their paralesional normal counterparts, LGIN-N, HGIN-N and CA-N, to estimate the differential expression of miR-92a. Statistical significance was defined as the two-tailed $\mathrm{P}$-value for rejecting the hypothesis of zero correlation and indicated using $\mathrm{P}<0.05$. A scatter diagram was plotted by Graphpad Prism ${ }^{\circledR}$ (Version 5.0; GraphPad Software, Inc., La Jolla, CA, USA) to illustrate the association between the visual grading of ISH and the OD value obtained from ISH-MSI.

\section{Results}

miR-92a expression level is visually indeterminable by ISH in CA, LGIN, HGIN and each of their corresponding paralesional normal controls. All tissue sections indicated at least partial positivity for miR-92a expression. Therefore, no section was graded as negative in Fig. 3. The median visual grading (VDx) of staining intensity was moderate for CA, CA-N, LGIN and LGIN-N, while for HGIN and HGIN-N the VD $x$ was moderate to positive and moderate, respectively.

miR-92a expression level significantly differs between $C A$, LGIN, HGIN and their paralesional normal controls, as determined by MSI analysis. Using the MSI system, the expression level of miR-92a in the cytoplasm was isolated 


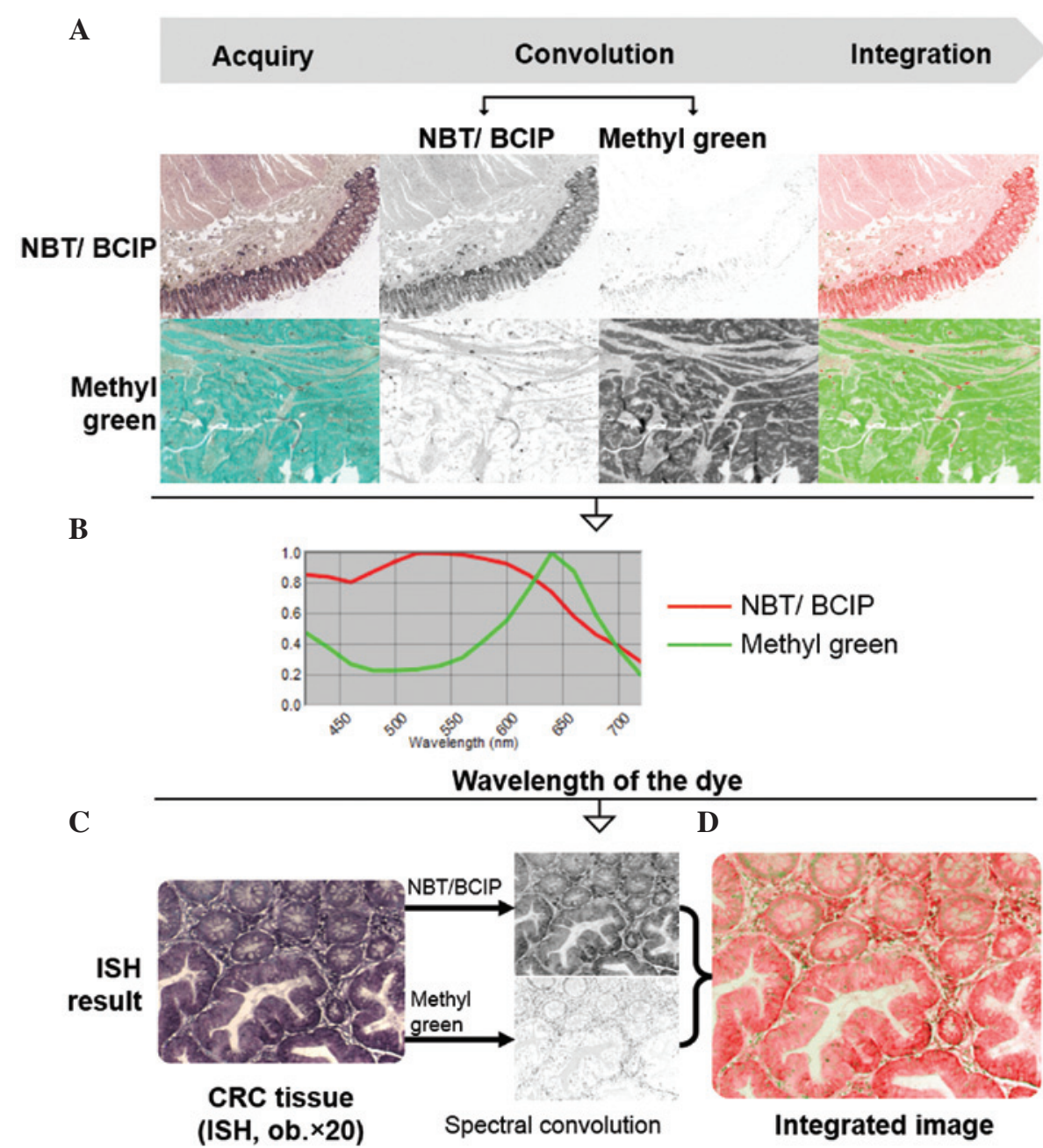

Figure 1. Principle of multispectral imaging technology in analyzing the expression level of miRNA. (A and B) Applying the CRi-Nuance ${ }^{\mathrm{TM}}$ Multispectral Imaging System. Dyes with a wavelength between 420 and $720 \mathrm{~nm}$ (NBT/BCIP and methyl green) were acquired as references (microphotographs at x50 magnification) and (C) microphotographs of tissues that underwent ISH, including colorectal adenocarcinoma (CA) tissues, were spectrally resolved to detect the expression level of miR-92a (magnification, x200). NBT/BCIP, nitro blue tetrazolium/5-bromo-4-chloro-3-indolyl phosphate; ISH, in situ hybridization; $\mathrm{miR} / \mathrm{miRNA}$, microRNA.

and further analyzed following the aforementioned calculations. Statistically significant differences were observed in the expression level of miR-92a between various categories of CA, CA-N, LGIN and HGIN, and their paralesional normal controls, LGIN-N and HGIN-N, respectively. In particular, for the average pixel area of the cytoplasm covered with NBT/BCIP, which determined the expression area of miR-92a, a significant difference was revealed when $\mathrm{CA}$ was compared with CA-N $(\mathrm{P}=0.020)$ and LGIN $(\mathrm{P}=0.018)$, when HGIN was compared with CA-N $(\mathrm{P}=0.027)$ and LGIN $(\mathrm{P}=0.018)$, when LGIN-N was compared with HGIN $(\mathrm{P}=0.012)$ and $\mathrm{CA}$ $(\mathrm{P}=0.0014)$, and when HGIN-N was compared with LGIN-N $(\mathrm{P}=0.0009)$. In the nuclei, the average pixel area of NBT/BCIP presented a significant difference in miR-92a expression when LGIN-N was compared with CA-N $(\mathrm{P}=0.019)$ and HGIN-N $(\mathrm{P}=0.031)$, when $\mathrm{CA}$ was compared with $\mathrm{CA}-\mathrm{N}(\mathrm{P}=0.0099)$, when CA-N was compared with LGIN $(\mathrm{P}=0.004)$ and when HGIN-N was compared with CA $(\mathrm{P}=0.014)$ and LGIN $(\mathrm{P}=0.006)$. For the expression intensity of miR-92a, denoted by NBT/BCIP, a significant difference was revealed when LGIN was compared with HGIN-N $(\mathrm{P}=0.012)$ and when CA-N was compared with $\operatorname{LGIN}(\mathrm{P}=0.0006)$. For the nuclei to cytoplasm ratio of miR-92a expression intensity, a significant difference was revealed when HGIN-N was compared with CA ( $\mathrm{P}=0.017)$, LGIN $(\mathrm{P}=0.038)$ and LGIN-N $(\mathrm{P}=0.046)$. Also, there was a significant difference when $\mathrm{CA}-\mathrm{N}$ was compared with HGIN $(\mathrm{P}=0.028)$. For the expression area of miR-92a over the ROI, a significant difference was revealed when HGIN-N was compared with $\mathrm{CA}(\mathrm{P}=0.032)$, LGIN $(\mathrm{P}=0.002)$ and LGIN-N ( $\mathrm{P}=0.011)$, when HGIN was compared with CA-N $(\mathrm{P}=0.032)$ and LGIN $(\mathrm{P}=0.011)$, when $\mathrm{CA}$ was compared with CA-N ( $\mathrm{P}=0.005)$, and when $\mathrm{CA}-\mathrm{N}$ was compared with LGIN $(\mathrm{P}=0.001)$ and LGIN-N $(\mathrm{P}=0.003)$. All these data are summarized and extrapolated in Fig. 4.

ISH combined with MSI legibly evaluates changes of miR-92a expression level in CA, LGIN, HGIN and their paralesional normal controls. The visual grading of the ISH results from the 34 samples was compared with the OD obtained by the combination of ISH and MSI in order to determine whether this modified technique could better evaluate the change in miR-92a expression. A scatter diagram plotted the visual grading of ISH (x-axis) against the OD value obtained from ISH-MSI (y-axis), as shown in Fig. 5. Linear regression 


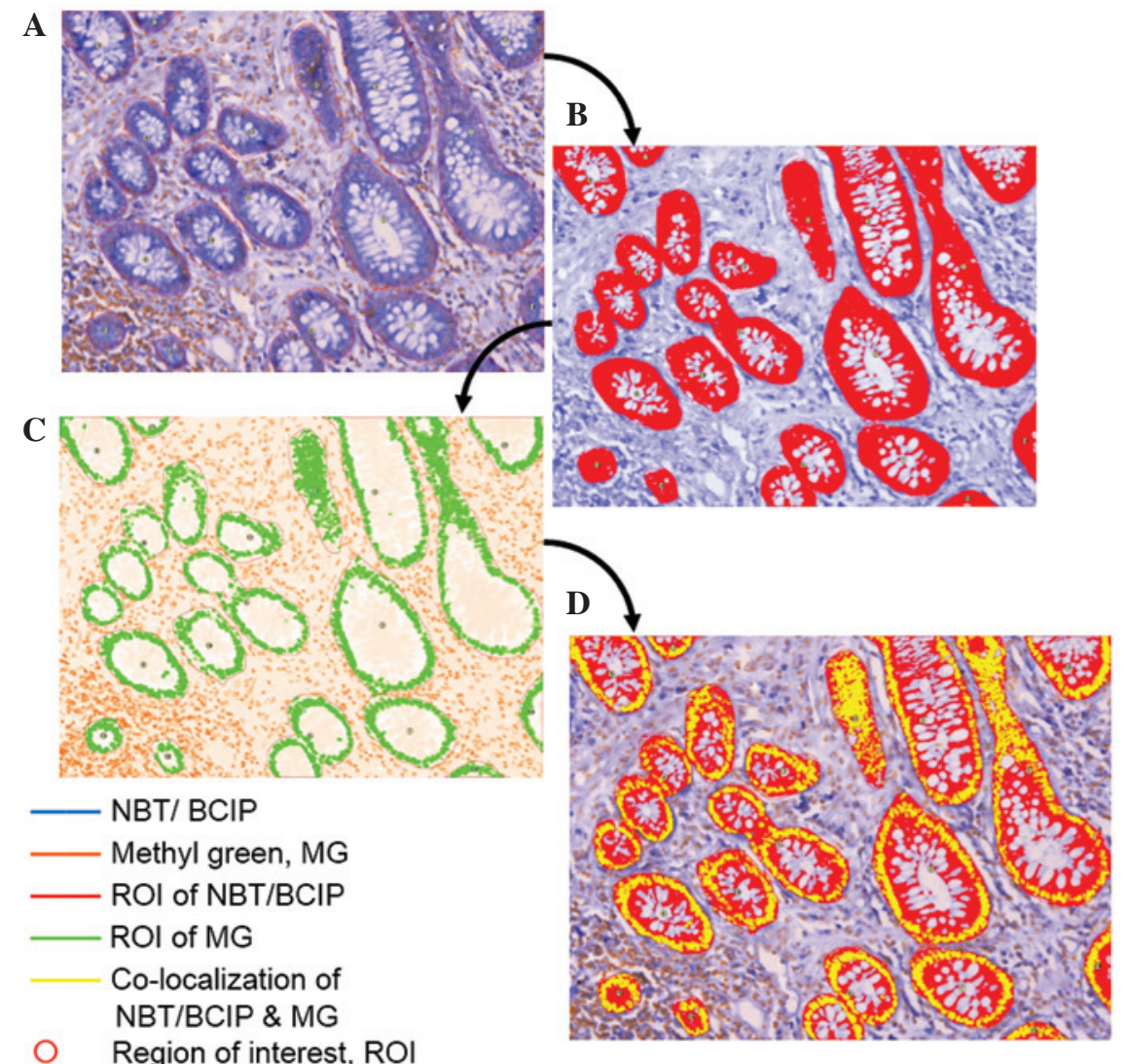

Figure 2. CRi-Nuance ${ }^{\mathrm{TM}}$ Multispectral Imaging System was applied to analyze the expression level of miR-92a in colorectal adenocarcinoma and its precancerous lesions. Applying the function of co-localization of Nuance to analyze the expression level of miR-92a in colorectal tissue, the expression pattern and intensity were then generated as raw data for further statistical assay. (A) A different color was appointed to represent NBT/BCIP and MG, and the region of interest was circled. In the image, blue represents NBT/ BCIP, orange represents MG and red circles represent the region of interest. (B) The threshold of ROI was adjusted and the orange color represents the area where miR-92a is expressed. (C) The threshold of MG was adjusted and the green color represents the area stained with the dye. (D) The threshold of the channel was adjusted, indicating co-localization of NBT/BCIP and MG. The yellow color represents data generated based on the pixel area, followed by calculation of the concentration of NBT/BCIP in the cytoplasm with the formula as provided, which represented the expression of miR-92a. NBT/BCIP, nitro blue tetrazolium/5-bromo-4-chloro-3-indolyl phosphate; ROI, region of interest; MG, methyl green.
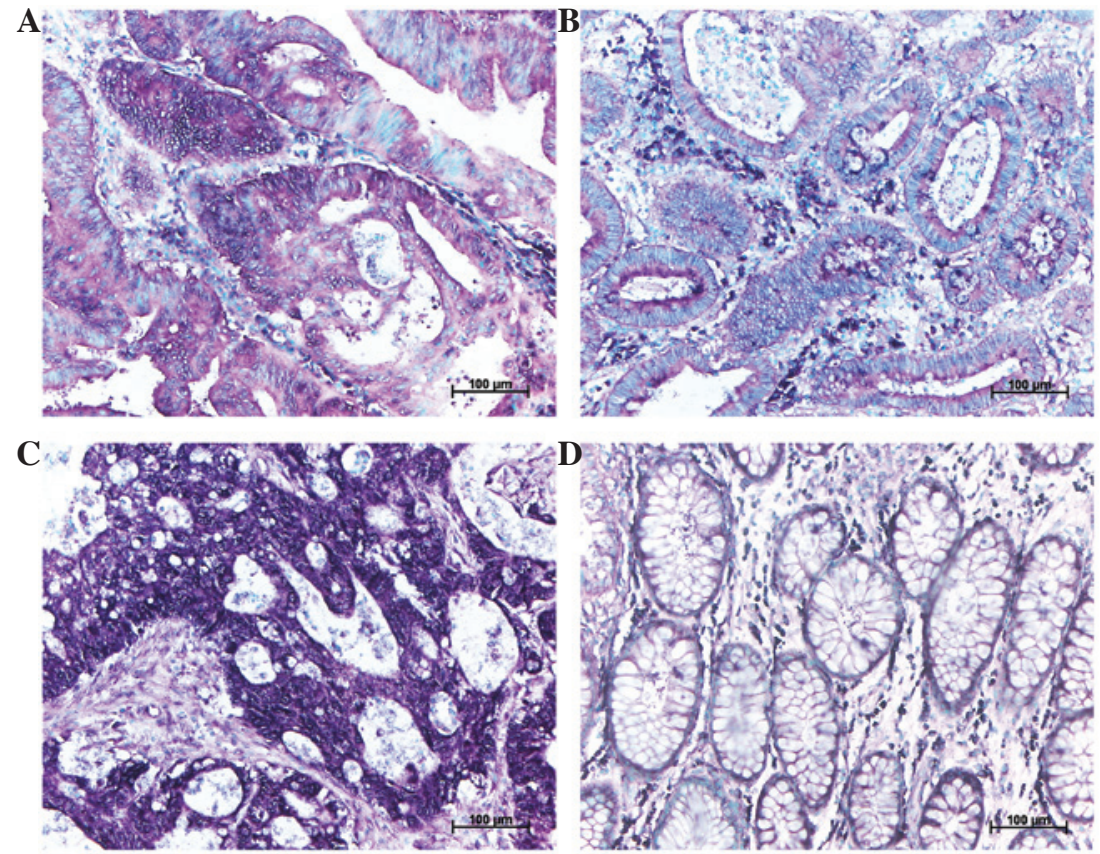

Figure 3. Expression of miR-92a in colorectal adenocarcinoma (CA) and its precancerous lesions, revealed by in situ hybridization (magnification, x200). (A) High-grade intraepithelial neoplasia. (B) Low-grade intraepithelial neoplasia. (C) CA. (D) Normal control of CA. Positive expression of miR-92a is represented by nitro blue tetrazolium/5-bromo-4-chloro-3-indolyl phosphate, with the nuclei counterstained by methylgreen. Scale bar, $100 \mu \mathrm{m}$. 
A

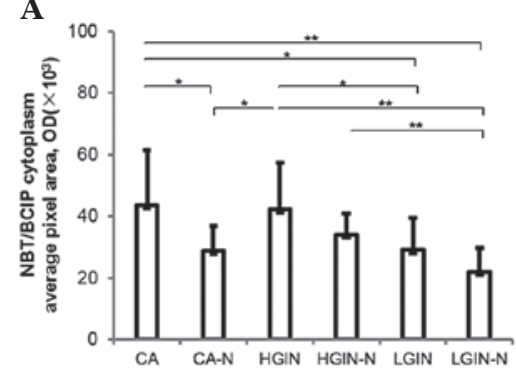

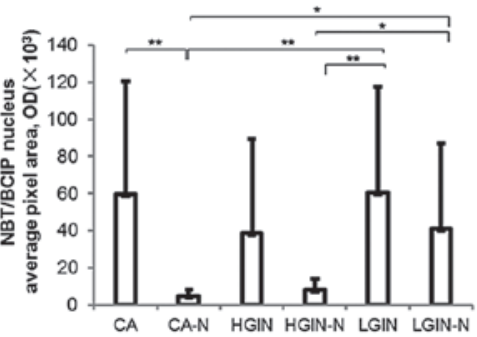

C

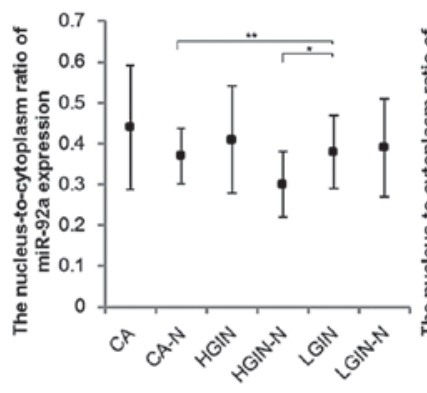

D

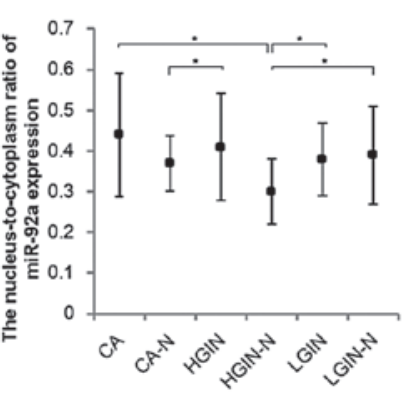

$\mathbf{E}$

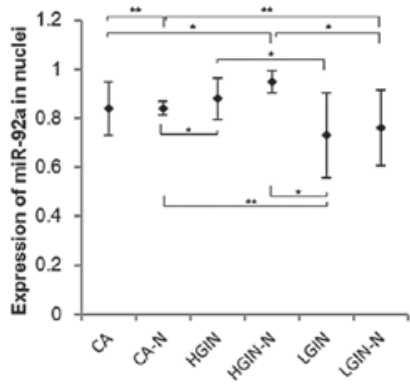

Figure 4. Multispectral imaging analysis of the expression level of miR-92a in 34 samples of colorectal adenocarcinoma (CA) and its precancerous lesions (CA, $\mathrm{n}=13$; CA-N, n=12; HGIN, n=11; HGIN-N, n=10; LGIN, n=10; and LGIN-N, n=9). (A) The average expression level of miR-92a in the cytoplasm of the tissue, determined by pixel area. (B) The average expression level of miR-92a in the nuclei of the tissue, determined by pixel area. (C) The average expression intensity of miR-92a in the cytoplasm of the tissue, determined by OD. (D) The nucleus-to-cytoplasm ratio of miR-92a expression in each group. (E) The expression of miR-92a in the nuclei of each group. The above data is calculated by the formula mentioned in the Materials and methods section. Each sample was repeated three times and the error bar represents the standard deviation. ${ }^{*} \mathrm{P}<0.05 ;{ }^{* *} \mathrm{P}<0.01$. CA, colorectal carcinoma; CA-N, CA paralesional normal tissue; HGIN, high-grade intraepithelial neoplasia; HGIN-N, HGIN paralesional normal tissue; LGIN, low-grade intraepithelial neoplasia; LGIN-N, LGIN paralesional normal tissue; NBT/ BCIP, nitro blue tetrazolium/5-bromo-4-chloro-3-indolyl phosphate; OD, optical density.

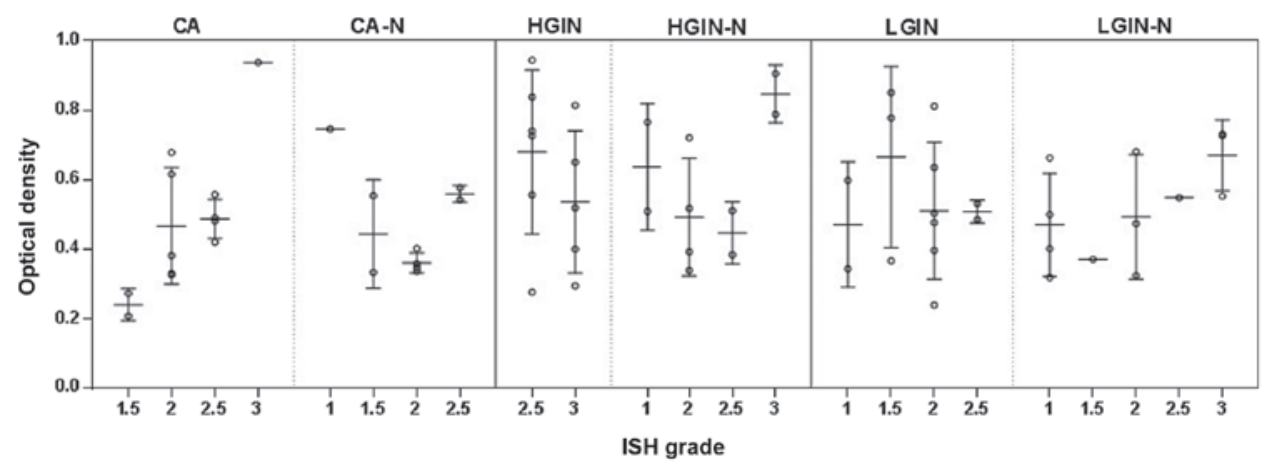

Figure 5. Comparison of stain intensity between spectral imaging (optical density) and visual grading (ISH grade) for all samples. All values were plotted and are shown as the mean \pm standard deviation (horizontal line). No intra- or inter-group colinearity was observed. CA, colorectal carcinoma; CA-N, CA paralesional normal tissue; HGIN, high-grade intraepithelial neoplasia; HGIN-N, HGIN paralesional normal tissue; LGIN, low-grade intraepithelial neoplasia; LGIN-N, LGIN paralesional normal tissue; ISH, in situ hybridization.

analysis revealed no significant correlation between the two quantification methods ( $\mathrm{rs}=0.25 ; \mathrm{P}>0.05$ ).

\section{Discussion}

The latest development of miRNA quantitation techniques initially focused on the cellular level to the body fluids, and included quantitative PCR assays $(18,19)$, next-generation sequencing (21), MSI (20) and miRNA sensing in living cells based on peptide nucleic acid and nano-graphene oxide (21). Until recently, no concrete method was designed to directly observe changes in the expression of miRNAs through immediate tissue observation. In several studies, the miR-92a expression level has been revealed to be elevated during the progression of CA (22-24). The hybridization signal on tissue sections did not provide a similar conclusion, as it revealed that miR-92a is universally, but unevenly, expressed in all studied samples, in CA, LGIN, HGIN and their paralesional normal controls. Nevertheless, this finding was consistent with the results of the study by Liang et al, which noted the expression of miR-92a in normal and cancerous organs (15). Compared with other common tumor indicators that are expressed in an all or nothing manner in cells, the expression characteristics of miR-92a resulted in an inconclusive, moderate, visual scoring for all studied samples containing colorectal lesions at various stages, with the exception of 
between HGIN and its normal control, inferring that an additional approach is required to evaluate the changes in the expression level of miR-29a.

Since the visual judgment of staining intensity was based on color development, MSI was recruited in the present study. MSI encompassed the ability to discriminate a wide color spectrum and evaluate the concentration of dye, as shown when this method was analogously applied in a study quantitating thymidylate synthase in CA (11). CISH was performed prior to imaging analysis, followed by the detection of expression level by the MSI system, using chromogens within the spectrum of 420 to $700 \mathrm{~nm}$ at the visible wavelength. The expression details, area and intensity, were calculated to precisely and objectively evaluate the differential expression of miR-92a from normal to cancerous colonic tissues. The results from quantitating the expression of miR-92a in CA, LGIN, HGIN and their paralesional normal controls with ISH-MSI revealed a significant difference between certain considered criteria, particularly the pattern and concentration of miR-92a expression in the nuclei and cytoplasm, assessed by the average pixel area and intensity from OD, respectively. The accretion of miR-92a from the precancerous lesions to CA by the nucleus-to-cytoplasm ratio and its amplitude of expression over the area studied demonstrated consistency with the findings of studies at the cellular, plasma and fecal levels (22-24), demonstrating that the observation of changes in the expression level of miRNA may be assessed at the microscopic level.

In the present study, objective visualization of the changes in miRNA expression was achieved with the implementation of CISH and MSI. ISH of miRNAs uses factitiously designed LNA-oligonucleotide probes that steadily and specifically detect minute concentrations of miRNA $(25,26)$. Likewise, hybridization using chromogens possesses several advantages compared with its fluorescence counterpart, as chromogen-labeling permits tangibility of specific cell types, which enables selection and observation of the region of interest for study under the light microscope, including the recognition of cancerous and normal colonic cells in the present study, since fluorophore-labeling techniques ambiguously discriminate between various cell types in the dark field. In addition, $\mathrm{CISH}$ requires a longer preservation period, with the generation of well-defined signals and the ability to select the definite regions of interest that radioactive and fluorescence counterparts cannot guarantee $(27,28)$. However, MSI demonstrates the capability of measuring multiple analytes carrying specific spectra at one time (29), which accurately resolves and relatively quantitates the changes in the miRNA expression level of various cell types, which conventional ISH would not achieve. This provides an objective and easy to use platform that is widely applied in a plethora of fields in biomedical research, including cytology, immunohistochemistry and nanoparticle studies $(7,8,30,31)$. Out of the available spectral imaging technology, the system employed in the present study used liquid crystal tunable filters, which confer advantages of a narrow spectral bandwidth, 7-20 nm, with an improved spectral resolution and a changeable wavelength at different ranges, such as the visible wavelength at 420-720 $\mathrm{nm}$ and near infra-red at 850-1,800 $\mathrm{nm}$, which allows evaluation for the fluorescence- and chromogen-based samples $(9,29)$. Coupling with a cooled scientific-grade monochrome CCD camera, the system produces excellent signal discrimination and image quality $(9,11)$.

Regardless of the frequent suggestions that the reproducibility of MSI could be undermined by artifacts generated during specimen preparation and the standardization of any parameters taken into the analysis, it has been suggested that, under strict and controlled conditions, artificial biases could be reduced to an optimal extent (32).

In conclusion, the co-utilization of ISH and spectral imaging analysis could validate the expression of miRNA through spectral and spatial evaluation, providing an improved understanding of its functions during the progression of diseases, generating valuable information for further study, in order to supply an effective and efficient diagnostic parameter for future clinical practice.

\section{References}

1. Wienholds E, Kloosterman WP, Miska E, et al: MicroRNA expression in zebrafish embryonic development. Science 309: 310-311, 2005.

2. Zhang B and Farwell MA: microRNAs: a new emerging class of players for disease diagnostics and gene therapy. J Cell Mol Med 12: 3-21, 2008.

3. Lu J, Getz G, Miska EA, et al: MicroRNA expression profiles classify human cancers. Nature 435: 834-838, 2005.

4. Yang L, Belaguli N and Berger DH: MicroRNA and colorectal cancer. World J Surg 33: 638-646, 2009.

5. Guiot Y and Rahier J: The effects of varying key steps in the non-radioactive in situ hybridization protocol: a quantitative study. Histochem J 27: 60-68, 1995.

6. Levsky JM and Singer RH: Fluorescence in situ hybridization: past, present and future. J Cell Sci 116: 2833-2838, 2003.

7. Levenson RM: Spectral imaging perspective on cytomics. Cytometry A 69: 592-600, 2006.

8. Barber PR, Vojnovic B, Atkin G, Daley FM, et al: Applications of cost-effective spectral imaging microscopy in cancer research. J Phys D: Appl Phys 36: 1729-1738, 2003.

9. Farkas DL, Du C, Fisher GW, et al: Non-invasive image acquisition and advanced processing in optical bioimaging. Comput Med Imaging and Graph 22: 89-102, 1998.

10. Levenson R, Beechem J and McNamara G: Spectral imaging in preclinical research and clinical pathology. Stud Health Technol Inform 185: 43-75, 2013.

11. Atkin G, Barber PR, Vojnovic B, et al: Correlation of spectral imaging and visual grading for the quantification of thymidylate synthase protein expression in rectal cancer. Hum Pathol 36: 1302-1308, 2005.

12. Slaby O, Svoboda M, Michalek J and Vyzula R: MicroRNAs in colorectal cancer: translation of molecular biology into clinical application. Mol Cancer 8: 102, 2009.

13. Ahmed FE, Jeffries CD, Vos PW, et al: Diagnostic microRNA markers for screening sporadic human colon cancer and active ulcerative colitis in stool and tissue. Cancer Genomics Proteomics 6: 281-295, 2009.

14. Wang S, Wang L, Bayaxi N, et al: A microRNA panel to discriminate carcinomas from high-grade intraepithelial neoplasms in colonoscopy biopsy tissue. Gut 62: 280-289, 2013.

15. Liang Y, Ridzon D, Wong L and Chen C: Characterization of microRNA expression profiles in normal human tissues. BMC Genomics 8: 166, 2007.

16. Jørgensen S, Baker A, Møller S and Nielsen BS: Robust one-day in situ hybridization protocol for detection of microRNAs in paraffin samples using LNA probes. Methods 52: 375-381, 2010.

17. Nuovo GJ, Elton TS, Nana-Sinkam P, et al: A methodology for the combined in situ analyses of the precursor and mature forms of microRNAs and correlation with their putative targets. Nat Protoc 4: 107-115, 2009.

18. Zhang Q, He XJ, Liu YJ, Ma LP and Pan XY: Profiling of microRNAs in mouse brain with real-time PCR array. Beijing Da Xue Xue Bao 41: 152-157, 2009 (In Chinese).

19. Chugh P, Tamburro K and Dittmer DP: Profiling of pre-micro RNAs and microRNAs using quantitative real-time PCR (qPCR) arrays. J Vis Exp 3: 2210, 2010. 
20. Mansfield JR: Cellular context in epigenetics: quantitative multicolor imaging and automated per-cell analysis of miRNAs and their putative targets. Methods 52: 271-280, 2010.

21. Ryoo SR, Lee J, Yeo J, et al: Quantitative and multiplexed microRNA sensing in living cells based on peptide nucleic acid and nano graphene oxide (PANGO). ACS Nano 7: 5882-5891, 2013.

22. Huang Z, Huang D, Ni S, et al: Plasma microRNAs are promising novel biomarkers for early detection of colorectal cancer. Int J Cancer 127: 118-126, 2010.

23. Ng EK, Chong WW, Jin H, et al: Differential expression of microRNAs in plasma of patients with colorectal cancer: a potential marker for colorectal cancer screening. Gut 58: 1375-1381, 2009.

24. Link A, Balaguer F, Shen Y, et al: Fecal MicroRNAs as novel biomarkers for colon cancer screening. Cancer Epidemiol Biomarkers Prev 19: 1766-1774, 2010.

25. Kloosterman WP, Wienholds E, de Bruijn E, Kauppinen S and Plasterk RH: In situ detection of miRNAs in animal embryos using LNA-modified oligonucleotide probes. Nat Methods 3: 27-29, 2006.

26. Stenvang J, Silahtaroglu AN, Lindow M, Elmen J and Kauppinen S: The utility of LNA in microRNA-based cancer diagnostics and therapeutics. Semin Cancer Biol 18: 89-102, 2008 .
27. Hara M, Yamada S and Hirata K: Nonradioactive In Situ Hybridization: Recent Techniques and Applications. Endocr Pathol 9: 21-29, 1998

28. Crabb ID, Hughes SS, Hicks DG, et al: Nonradioactive in situ hybridization using digoxigenin-labeled oligonucleotides. Applications to musculoskeletal tissues. Am J Pathol 141: 579-589, 1992

29. Ornberg RL, Woerner BM and Edwards DA: Analysis of stained objects in histological sections by spectral imaging and differential absorption. J Histochem Cytochem 47: 1307-1314, 1999.

30. Levenson RM and Hoyt CC: Spectral imaging and microscopy. Am Lab 32: 26-34, 2000

31. Kavvadias V, Epitropou G, Georgiou N, et al: A novel endoscopic spectral imaging platform integrating k-means clustering for early and non-invasive diagnosis of endometrial pathology. Conf Proc IEEE Eng Med Biol Soc 2013: 4442-4445, 2013.

32. Seidal T, Balaton AJ and Battifora $\mathrm{H}$ : Interpretation and quantification of immunostains. Am J Surg Pathol 25: 1204-1207, 2001. 